Bull. Austral. Math. Soc.

16A12, 16A70, 16A72

VoL. 53 (1995) [353-359]

\title{
DERIVATIONS ON SEMIPRIME RINGS
}

\section{Joso VUKMAN}

The main result: Let $R$ be a 2 -torson free semiprime ring and let $D: R \rightarrow R$ be a derivation. Suppose that $[[D(x), x], x]=0$ holds for all $x \in R$. In this case $[D(x), x]=0$ holds for all $x \in R$.

Throughout, $R$ will represent an associative ring with centre $Z(R)$. The commutator $x y-y x$ will be denoted by $[x, y]$. We make extensive use of the basic commutator identities $[x y, z]=[x, z] y+x[y, z],[x, y z]=[x, y] z+y[x, z]$. An additive mapping $D$ from $R$ to $R$ is called a derivation if $D(x y)=D(x) y+x D(y)$ holds for all $x, y \in R$. A derivation $D$ is inner if there exists $a \in R$ such that $D(x)=[a, x]$ holds for all $\mathrm{x} \in R$. Recall that $R$ is prime if $a R b=(0)$ implies that either $a=0$ or $b=0$, and is called semiprime in case $a R a=(0)$ implies $a=0$. A mapping $F$ of $R$ into itself is called centralising on $R$ if $[F(x), x] \in Z(R)$ holds for all $x \in R$; in the special case when $[F(x), x]=0$ holds for all $x \in R$, the mapping $F$ is said to be commuting on $R$. The study of centralising and commuting mappings was initiated by the classical result of Posner [6], which states that the existence of a nonzero centralising derivation on a prime ring forces the ring to be commutative (Posner's second theorem). A lot of work has been done during the last twenty years in this field (see $[2,3,4]$ where further references can be found). It is our aim in this paper to present some results which can be considered as a contribution to the theory of commuting and centralising mappings in semiprime rings.

Theorem 1. Let $R$ be a 2-torsion free semiprime ring. Suppose there exists a derivation $D: R \rightarrow R$, such that the mapping $x \mapsto[D(x), x]$ is commuting on $R$. In this case $D$ is commuting on $R$.

Proof: Let us introduce a mapping $B(.,):. R \times R \rightarrow R$ by the relation $B(x, y)=$ $[D(x), y]+[D(y), x], x, y \in R$. Obviously, $B(.,$.$) is symmetric (that is B(x, y)=$ $B(y, x)$ for all $x, y \in R)$ and additive in both arguments. A routine calculation shows that the relation

$$
B(x y, z)=B(x, z) y+x B(y, z)+D(x)[y, z]+[x, z] D(y)
$$

Received 15 June 1995

This research has been supported by the Research Council of Slovenia.

Copyright Clearance Centre, Inc. Serial-fee code: 0004-9729/95 \$A2.00+0.00. 
holds for all $x, y, z \in R$. We introduce also a mapping $f$ from $R$ to $R$ by $f(x)=$ $B(x, x)$. We have $f(x)=2[D(x), x], x \in R$. The mapping $f$ satisfies the relation

$$
f(x+y)=f(x)+f(y)+2 B(x, y), \quad x, y \in R .
$$

Throughout the paper we shall use the mappings $B$ and $f$, as well as the relations (1) and (2) without specific references. The assumption of the theorem can now be written in the form

$$
[f(x), x]=0, x \in R
$$

The linearisation of (3) gives

$$
[f(x), y]+[f(y), x]+2[B(x, y), x]+2[B(x, y), y]=0, \quad x, y \in R .
$$

Substituting in the above relation $x$ by $-x$ and comparing the relation so obtained with the above relation we arrive at

$$
[f(x), y]+2[B(x, y), x]=0, \quad x, y \in R .
$$

Replace $y$ in (4) by $x y$. Then $0=[f(x), x y]+2[B(x y, x), x]=[f(x), x y]+$ $2[f(x) y+x B(x, y)+D(x)[y, x], x]=[f(x), x] y+x[f(x), y]+2[f(x), x] y+2 f(x)[y, x]+$ $2 x[B(x, y), x]+f(x)[y, x]+2 D(x)[[y, x], x]$, which reduces to

$$
3 f(x)[y, x]+2 D(x)[[y, x], x]=0, \quad x, y \in R,
$$

according to (3) and (4). In the same fashion one obtains

$$
3[y, x] f(x)+2[[y, x], x] D(x)=0, \quad x, y \in R,
$$

replacing $y$ in (4) by $y x$. Let us prove the relation

$$
3 f(x) D(x)=D(x) f(x), \quad x \in R .
$$

For this purpose we substitute (5) $y z$ for $y$ in (5). Then $0=3 f(x)[y z, x]+$ $2 D(x)[[y z, x], x]=3 f(x)[y z, x]+2 D(x)[[y, x] z+y[z, x], x]=3 f(x)[y, x] z+3 f(x) y[z, x]+$ $2 D(x)[[y, x], x] z+4 D(x)[y, x][z, x]+2 D(x) y[[z, x], x]$, whence it follows that

$$
3 f(x) y[z, x]+4 D(x)[y, x][z, x]+2 D(x) y[[z, x], x]=0, \quad x, y, z \in R,
$$

and in particular for $y=D(x), z=y$

$$
3 f(x) D(x)[y, x]+2 D(x) f(x)[y, x]+2 D(x)^{2}[[y, x], x]=0, \quad x, y \in R .
$$


Left multiplication of (5) by $D(x)$ gives

$$
3 D(x) f(x)[y, x]+2 D(x)^{2}[[y, x], x]=0, \quad x, y \in R .
$$

From (9) and (10) it follows that $(3 f(x) D(x)-D(x) f(x))[y, x]=0, \quad x, y \in R$, which gives

$$
(3 f(x) D(x)-D(\quad x) f(x)) y[z, x]=0 . \quad x, y, z \in R
$$

By putting $z=2 D(x)$ in (11) we obtain

$$
(3 f(x) D(x)-D(x) f(x)) y f(x)=0, \quad x, y \in R .
$$

Right multiplication of (12) by $3 D(x)$ gives

$$
(3 f(x) D(x)-D(x) f(x)) y 3 f(x) D(x)=0, \quad x, y \in R .
$$

Putting $y D(x)$ for $y$ in (12) we arrive at

$$
(3 f(x) D(x)-D(x) f(x)) y D(x) f(x)=0, \quad x, y \in R .
$$

By subtracting (14) from (13) we obtain

$$
(3 f(x) D(x)-D(x) f(x)) y(3 f(x) D(x)-D(x) f(x))=0, \quad x, y \in R
$$

which proves (7) by semiprimeness of $R$. In the same fashion one obtains

$$
3 D(x) f(x)=f(x) D(x), \quad x \in R,
$$

starting from (6). From (15) and (16) it follows immediately

$$
f(x) D(x)=0, \quad x \in R,
$$

and

$$
D(x) f(x)=0, \quad x \in R
$$

From (17) one obtains easily

$$
f(x) D(y)+2 B(x, y) D(x)=0, \quad x, y \in R
$$

By substituting $x y$ for $y$ in (19) we obtain

$$
\begin{aligned}
0 & =f(x) D(x y)+2 B(x y, x) D(x) \\
& =f(x) D(x) y+f(x) x D(y)+2 f(x) y D(x)+2 x B(x, y) D(x)+2 D(x)[y, x] D(x)
\end{aligned}
$$


According to (18) we can write $-f(x) D(y)$ instead of $2 B(x, y) D(x)$ in the above calculation which, gives $[f(x), x] D(y)+2 f(x) y D(x)+2 D(x)[y, x] D(x)=0$ whence it follows that

$$
f(x) y D(x)+D(x)[y, x] D(x)=0, \quad x, y \in R,
$$

according to (3). Replacing in (20) $y$ by $y x$ we obtain

$$
f(x) y x D(x)+D(x)[y, x] x D(x)=0, \quad x, y \in R .
$$

Right multiplication of (20) by $x$ gives

$$
f(x) y D(x) x+D(x)[y, x] D(x) x=0, \quad x, y \in R .
$$

By subtracting (21) from (22) we obtain

$$
f(x) y f(x)+D(x)[y, x] f(x)=0, \quad x, y \in R .
$$

By putting $z=2 D(x)$ in (8), we obtain

$$
3 f(x) y f(x)+4 D(x)[y, x] f(x)=0, \quad x, y \in R .
$$

From (23) and (24) it follows that $f(x) y f(x)=0, x, y \in R$, whence $[D(x), x]=0$, $x \in R$. The proof of the theorem is complete.

In our earlier paper [7] one can find an extension of Posner's second theorem which states that there is no nonzero derivation on a noncommutative prime ring of characteristic different from two satisfying the relation $[[D(x), x], x]=0$ for all $x \in R$. This result has been generalised by Brešar [2] and Lanski [5]. Since in noncommutative semiprime rings there exist nonzero commuting derivations, the assumptions of Theorem 1 do not imply that $D=0$. However, in the special case when $D$ is an inner derivation, one can prove the following result.

COROLlary 2. Let $R$ be a 2-torsion free noncommutative semiprime ring. Suppose there exists an inner derivation $D: R \rightarrow R$, such that the mapping $x \mapsto[D(x), x]$ is commuting on $R$. In this case $D=0$.

Proof: An immediate consequence of Theorem 1 and Corollary 5 in [1].

We continue with the following result.

THEOREM 3. Let $R$ be a 2-torsion free and 3-torsion free semiprime ring and $D: R \rightarrow R$ a derivation. Suppose that the mapping $x \mapsto[D(x), x]$ is centralising on $R$. In this case $D$ is commuting on $R$.

The result above is not a generalisation of Theorem 1 , since in Theorem 3 we have an additional assumption that $R$ is 3 -torsion free. We feel that this assumption can be avoided, but unfortunately we were unable to do it. 
Proof of Theorem 3: According to the assumption of the theorem we have

$$
[f(x), x] \in Z(R), \quad x \in R .
$$

Similarly, as in the proof of identity (4) one can prove that

$$
[f(x), y]+2[B(x, y), x] \in Z(R), \quad x, y \in R .
$$

The substitution $y=x^{2}$ in (16) gives $\left[f(x), x^{2}\right]+2[f(x) x+x f(x), x] \in Z(R)$, and $[f(x), x] x+x[f(x), x]+2[f(x), x]+2[f(x), x] x+2 x[f(x), x] \in Z(R)$. Thus we have

$$
6[f(x), x] x \in Z(R), \quad x \in R .
$$

From (24) and (26) it follows $6[f(x), x][y, x]=0, x, y \in R$, which gives

$$
[f(x), x][y, x]=0, x, y \in R,
$$

since we have assumed that $R$ is 2-torsion free and 3-torsion free. By putting $y f(x)$ for $y$ in the above relation we obtain

$$
[f(x), x] y[f(x), x]=0, x, y \in R,
$$

whence it follows that $[f(x), x]=0, x \in R$ which completes the proof of the theorem since all the requirements of Theorem 1 are fulfilled.

Our next result was inspired by Posner's first theorem [6], which asserts that if $R$ is a prime ring of characteristic different from two, and $D, G$ are nonzero derivations on $R$, then $D G$ cannot be a derivation.

THEOREM 4. Let $R$ be a 2-torsion free semiprime ring and $D: R \rightarrow R, G: R \rightarrow$ $R$ derivations. Suppose that the mapping $x \mapsto D^{2}(x)+G(x)$ is centralising on $R$. In this case $D$ and $G$ are both commuting on $R$.

Proof of Theorem 4: By Proposition 3.1 in [4], the mapping $x \mapsto D^{2}(x)+G(x)$ is commuting on $R$. Thus we have

$$
[F(x), x]=0, \quad x \in R,
$$

where $F(x)$ stands for $D^{2}(x)+G(x)$. The linearisation of (28) leads to

$$
[F(x), y]+[F(y), x]=0, \quad x, y \in R .
$$

By substituting for $y$ in (29) $y x$ and noting that

$$
F(y x)=F(y) x+y F(x)+2 D(y) D(x), \quad x, y \in R,
$$


we then obtain

$$
\begin{aligned}
0= & {[F(x), y x]+[F(y x), x]=[F(x), y x]+[F(y) x+y F(x)+2 D(y) D(x), x] } \\
= & {[F(x), y] x+y[F(x), x]+[F(y), x] x+[y, x] F(x)+y[F(x), x] } \\
& +2[D(y), x] D(x)+2 D(y)[D(x), x] .
\end{aligned}
$$

According to (28) and (29) the above relation reduces to

$$
[y, x] F(x)+2[D(y), x] D(x)+2 D(y)[D(x), x]=0, \quad x, y \in R
$$

By replacing $y$ by $x y$ in (30) we obtain

$$
\begin{aligned}
0= & {[x y, x] F(x)+2[D(x y), x] D(x)+2 D(x y)[D(x), x] } \\
= & {[x y, x] F(x)+2[D(x) y+x D(y), x] D(x)+2 D(x y)[D(x), x] } \\
= & x[y, x] F(x)+2[D(x), x] y D(x)+2 D(x)[y, x] D(x)+2 x[D(y), x] D(x) \\
& +2 D(x) y[D(x), x]+2 x D(y)[D(x), x] .
\end{aligned}
$$

Using (30) it follows from the above calculation that

$$
[D(x), x] y D(x)+D(x)[y, x] D(x)+D(x) y[D(x), x]=0, \quad x, y \in R
$$

Let us replace $y$ by $y D(x) z$ in (31). Then

$$
\begin{aligned}
0= & {[D(x), x] y D(x) z D(x)+D(x)[y D(x) z, x] D(x)+D(x) y D(x) z[D(x), x] } \\
= & {[D(x), x] y D(x) z D(x)+D(x)[y, x] D(x) z D(x)+D(x) y[D(x), x] z D(x) } \\
& +D(x) y D(x)[z, x] D(x)+D(x) y D(x) z[D(x), x]
\end{aligned}
$$

which because of $(31)$ reduces to $D(x) y D(x)[z, x] D(x)+D(x) y D(x) z[D(x), x]=0$, and using again (31) to

$$
D(x) y[D(x), x] z D(x)=0, \quad x, y, x \in R
$$

Right multiplication of the above relation by $y[D(x), x]$ give

$$
D(x) y[D(x), x] z D(x) y[D(x), x]=0, \quad x, y, x \in R
$$

whence it follows that

$$
D(x) y[D(x), x]=0, \quad x, y \in R
$$


because of the semiprimeness of $R$. By replacing $y$ by $x y$ in (32) we obtain

$$
D(x) x y[D(x), x]=0, \quad x, y \in R .
$$

Left multiplication of (32) by $x$ gives

$$
x D(x) y[D(x), x]=0, \quad x, y \in R .
$$

From (33) and (34) it follows that

$$
[D(x), x] y[D(x), x]=0, \quad x, y \in R
$$

which means that

$$
[D(x), x]=0, \quad x \in R .
$$

The proof of the first part of the theorem is complete. The linearisation of (35) gives $[D(x), y]+[D(y), x]=0, x, y \in R$ anci in particular for $y=D(x)$

$$
\left[D^{2}(x), x\right]=0, \quad x \in R \text {. }
$$

Using the above relation in (28) we obtain that $[G(x), x]=0, x \in R$, which completes the proof of the theorem.

The result below is an immediate consequence of Theorem 4 and Corollary 5 in $[1]$.

Corollary 5. Let $R$ be a 2-torsion free noncommutative semiprime ring and let $D: R \rightarrow R, G: R \rightarrow R$ be derivations. Suppose that the mapping $x \mapsto D^{2}(x)+G(x)$ is centralising on $R$. If $D$ is inner we have that $D=0$. If $G$ is inner then $G=0$.

\section{REFERENCES}

[1] M. Brešar and J. Vukman, 'Orthogonal derivation and an extension of a theorem of Posner', Rad Mat. 5 (1989), 237-246.

[2] M. Brešar, 'On a generalization of the notion of centralising mappings', Proc. Amer. Math. Soc. 114 (1992), 641-649.

[3] M. Brešar, 'Commuting traces of biaditive mappings, commutativity-preserving mappings and Lie mappings', Trans. Amer. Math. Soc. 335 (1993), 525-546.

[4] M. Brešar, 'Centralizing mappings and derivations in prime rings', J. Algebra 156 (1993), 385-394.

[5] C. Lanski, 'An Engel condition with derivations', Proc. Amer. Math. Soc. 118 (1993), 731-734.

[6] E.C. Posner, 'Derivations in prime rings', Proc. Amer. Math. Soc. 8 (1957), 1093-1100.

[7] J. Vukman, 'Commuting and centralizing mappings in prime rings', Proc. Amer. Math. Soc. 109 (1990), 47-52.

Department of Mathematics

University of Maribor

PF, Koroška 160

62000 Maribor

Slovenia 